\title{
Algorithmic Approaches For Image Edge Detection
}

\author{
${ }^{1}$ Afrozah Nadeem, ${ }^{2}$ SyedaBinish Zahra, ${ }^{3}$ Sundus Munir, ${ }^{4}$ Sadia Kousar, ${ }^{5}$ Arfa Hassan, ${ }^{6}$ Abdul Rehman \\ Lahore Garrison University, Department of Computer Science, Lahore, Pakistan ${ }^{1,2,3,4,5,6}$ \\ 19afrozah@1gu.edu.pk, ${ }^{2}$ binishzahra@lgu.edu.pk, ${ }^{3}$ sundusmunir@lgu.edu.pk, ${ }^{4}$ sadiakousar@lgu.edu.pk, \\ ${ }^{5}$ arfahassan@lgu.edu.pk, ${ }^{6}$ abdulrehman@lgu.edu.pk
}

\begin{abstract}
Edge detection is most important technique in digital image processing. It play an important role in image segmentation and many other applications. Edge detection provides foundation to many medical and military applications. It difficult to generate a generic code for edge detection so many kinds of algorithms are available. In this article 4 different approaches Global image enhancement with addition (GIEA),
\end{abstract}

\section{INTRODUCTION}

Digital image processing is one of the most important tasks as it helps us in all fields of life. Edge detection is a method in digital image processing which play vital role in many applications.As it is provide foundation to many image processing and computer vision applications[1].The main concept of edge detection is to get important information regarding internal or inside the image object. It helpful in medical field to detect the different disease and effected parts of body. Edge detection techniques is also beneficial in military application[2]. Through edge detection techniques system can check continues pixel intensity of digital images. Edge detection on noisy images is challenging task because inside image inside object is not clear. Edge detection is technique which is concern with localization of pixels [3].

Edge detection is complex and difficult task. A lot of work is done on this topic but researcher could not able to find any generic approach for edge detection because every digital image have different resolution and intensity[4].Many kinds of algorithm are available which shows the importance of researchers in this topic. Edge detection is play an important role in object detection and biometrics applications[5].In this article 4 different algorithms are proposed for edge detection system. These are as following:
- GIEA
- GIEM
- WOGIEA
- WOGIEM

Global image enhancement with Multiplication (GIEM),Without Global image enhancement with Addition (WOGIEA), and without Global image enhancement with Multiplication (WOGIEM) for edge detection is proposed. These algorithms are validated on 9 different images. The results show that GIEA give us more accurate results as compare to other techniques.

Key words: Edge detection, Digital image processing, WOGIEA, WOGIEM, GIEA, GIEM

GIEA and WOGIEA is shows more efficient results as compare to GIEM and WOGIMA techniques. These algorithms are design by using convolution methods. For this purpose, GIEA and GIEM are convolve with enhance image and other algorithms are convolved with original image.

\section{RELATED WORK}

In digital image processing history researchers introduce many application and algorithms of edge detection but a few of them are discuss in this section.

In [4], [6] luminous density based algorithm is proposed for edge The beneficial of this algorithms is much faster as compare to the other techniques. In other work reberts and prewitt algorithms are proposed. Prwit and reberts focus on edge intensity of light and relationship among them[7]. [8]sober and Robert are widely used edge detection algorithms.In these edge detection algorithm intensity y $(\mathrm{x}, \mathrm{y})$ and it reflection $\mathrm{g}(\mathrm{x}, \mathrm{y})$ are used. The formula purposed is as following.

Orientation Edge: in which the most sensitive edge of image is determine by operator of geometry for looking optimize in different direction like vertical horizontal or diagonal.

Noise environment: it is consisting of different shape of images like noisy pixel blurred result. 
Edge structure: it is not to be change intensity effects like as refraction and not good focus for efficient result for excellent time and different location.

Sobel and Robert clos technique used first derivate has very simple calculation bit it disadvantages inaccurate detection[9]. [10] 3D prewitt and sobal algorithms are introduced for edge detection in 3D images. But in [11]prewitt, sobal and canny edge detection algorithms are apply on FGPA.In FPGA environment prewitt edge detection algorithm is gives better results as compare to sobal and canny edge detection algorithm [16].

Edge detection in ship infrared images is challenging task. Because these images are not clear due to fog, water [17] and light interface. For this purpose in [12] improved adaptive canny edge detection algorithm is proposed. Edge detection also play an important role in robotics. In article [13] a edge detection algorithm is introduced which is used in robot path planning.[14] In other work the researcher introduced Otsu-Canny Operator edge detection algorithm for Parallel Image Edge Detection on the Hadoop Platform. In other work the scientist introduces the edge detection methods for steganography. A lot of method already exit steganography, but the edge detection algorithm gives the best results. So the research introduced LSBMR approach[8], [15].

\section{PROPOSED METHOD}

In this research article four different algorithms are proposed for edge detection and discuss about the performance of these proposed methods. These proposed algorithms are as following.

- GIEA (Global image enhancement with Addition)

- GIEM (Global image enhancement with Multiplication)

- WOGIEM (Without Global image enhancement with Multiplication)

- WOGIEA (Without Global image enhancement with Addition)

A few initial steps of all these proposed algorithms are some. The detail flow chart of these algorithms is shown in figure 1.

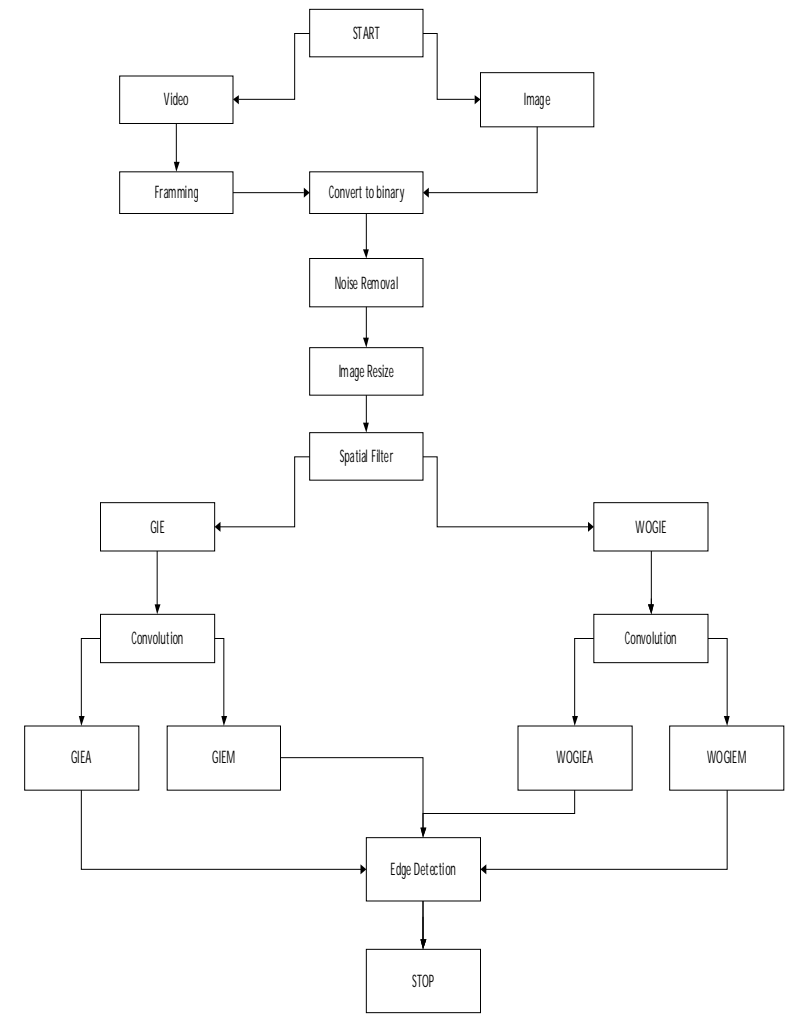

Figure 1

Figure 1 shows that, system acquire the data and verify that is image or video. If the acquire data is in the form of video, then algorithm convert it in the form of farms and move towards the next step. After that, the proposed algorithm converts the image into binary image and move to next step. After the noise removal and resizing the algorithm apply spatial filter [19].

At this level all proposed algorithms are work uniquely. First proposed algorithm GIEA perform histogram equalization for image enhancement then deconvolve after that image is convolve with equalized image and then add both $\mathrm{x}$ coordinates and y coordinates image by using matrix addition but in GIEM both images are merge by using matrix multiplication technique. In the next 2 algorithm WOGIEM and WOGIEA is the convolution is performed with original images instead of equalized images. [20]The mathematical explanation and block diagram of these algorithmsare given below:

$\underline{\text { GIEA }}$ 


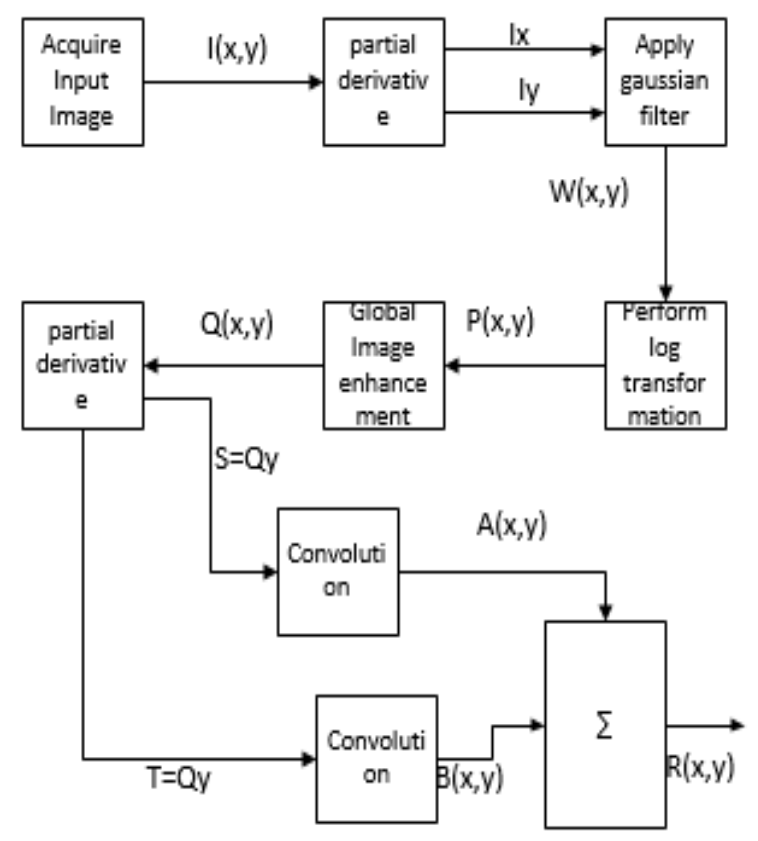

Figure 2

The acquire RGB image $I(\mathrm{x}, \mathrm{y})$ where we have to calculate $I_{\mathrm{x}}, \& I_{\mathrm{y}}$ from the Image $I(\mathrm{x}, \mathrm{y})$.

For the Further precedence:

Take partial derivative of the RGB image $I(\mathrm{x}, \mathrm{y})$ $\left[\begin{array}{l}I x \\ I y\end{array}\right]=\left[\begin{array}{c}\frac{\partial I(x, y)}{\partial x} \\ \frac{\partial I(x, y)}{\partial y}\end{array}\right]$

Apply Gaussian Filter to remove the noise from the images

Noise Removal: $W=\frac{1}{\sqrt{\sqrt{(2 \pi \sigma)}}} e^{-\frac{\left(I x^{2}+I y^{2}\right)}{2 \sigma}}$

Take logarithm

$\mathrm{P}=\log (\mathrm{W},(\mathrm{x}, \mathrm{y}))$

Calculate Histogram

$\mathrm{P}\left(\mathrm{r}_{\mathrm{k}}\right)=\mathrm{n}_{\mathrm{k}}$ where $\mathrm{k}=1 \ldots \ldots \ldots \mathrm{n}$

$\mathrm{Q}=(\mathrm{L}-1) \sum_{k=1}^{n} P(r k)$

Apply partial derivation

$\left[\begin{array}{l}Q x \\ Q y\end{array}\right]=\left[\begin{array}{c}\frac{\partial Q(x, y)}{\partial x} \\ \frac{\partial Q(x, y)}{\partial y}\end{array}\right]$

Apply convolution on both the imagaes

$S=Q(x)$

$T=Q(y)$

$\mathrm{g}(\mathrm{Q}, \mathrm{S})=\mathrm{Q}(\mathrm{x}, \mathrm{y}) * \mathrm{~S}(\mathrm{x}, \mathrm{y})$

$\mathrm{g}(\mathrm{Q}, \mathrm{S})=\sum_{u}^{a}=-a \sum_{v}^{b}=-b Q(u, v) S(x-u, y-v)$

$\mathrm{A}(\mathrm{x}, \mathrm{y})=\mathrm{g}(\mathrm{Q}, \mathrm{S})=\sum_{u}^{a}=-a \sum_{v}^{b}=-b Q(u, v) S(x-$

$u, y-v) \ldots \ldots \ldots . .1$

$\mathrm{G}(\mathrm{Q}, \mathrm{T})=\mathrm{Q}(\mathrm{x}, \mathrm{y}) * \mathrm{~T}(\mathrm{x}, \mathrm{y})$

$\mathrm{g}(\mathrm{Q}, \mathrm{T})=\sum_{u}^{a}=-a \sum_{v}^{b}=-b Q(u, v) T(x-u, y-v)$

$\mathrm{B}(\mathrm{x}, \mathrm{y})=\mathrm{g}(\mathrm{Q}, \mathrm{T})=\sum_{u}^{a}=-a \sum_{v}^{b}=-b Q(u, v) T(x-$

$u, y-v) \ldots . . . . .2$
From equation 1 and 2 add the results

$\mathrm{X}(\mathrm{x}, \mathrm{y})=\mathrm{A}(\mathrm{x}, \mathrm{y})+\mathrm{B}(\mathrm{x}, \mathrm{y})$

\section{$\underline{\text { GIEM }}$}

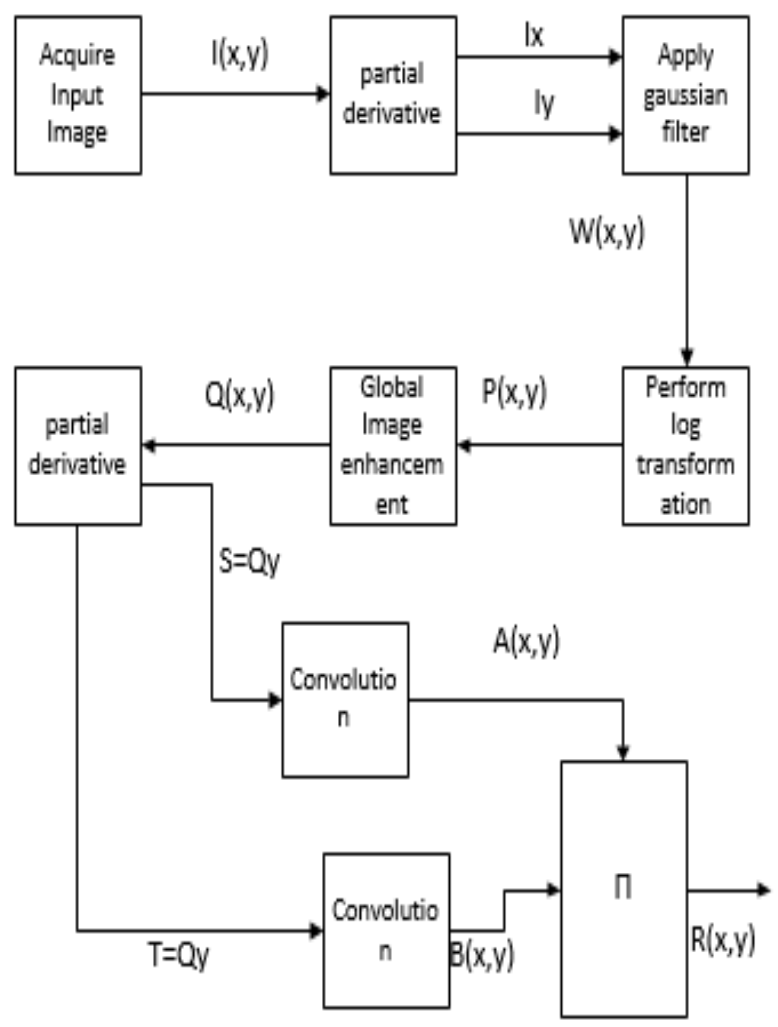

Figure 3

The acquire RGB image $I(\mathrm{x}, \mathrm{y})$ where we have to calculate $I_{\mathrm{x}}, \& I_{\mathrm{y}}$ from the Image $I(\mathrm{x}, \mathrm{y})$.

For the Further precedence:

$\left[\begin{array}{l}I x \\ I y\end{array}\right]=\left[\begin{array}{c}\frac{\partial I(x, y)}{\partial x} \\ \frac{\partial I(x, y)}{\partial y}\end{array}\right]$

Noise Removal: $W=\frac{1}{\sqrt{\sqrt{(2 \pi \sigma)}}} e^{-\frac{\left(I x^{2}+I y^{2}\right)}{2 \sigma}}$

$\mathrm{P}=\log (\mathrm{W},(\mathrm{x}, \mathrm{y}))$

Calculate Histogram

$\mathrm{P}\left(\mathrm{r}_{\mathrm{k}}\right)=\mathrm{n}_{\mathrm{k}}$ where $\mathrm{k}=1 \ldots \ldots \ldots \mathrm{n}$

$\mathrm{Q}=(\mathrm{L}-1) \sum_{k=1}^{n} P(r k)$

$\left[\begin{array}{l}Q x \\ Q y\end{array}\right]=\left[\begin{array}{c}\frac{\partial Q(x, y)}{\partial x} \\ \frac{\partial Q(x, y)}{\partial y}\end{array}\right]$

$S=Q(x)$

$T=Q(y)$

$\mathrm{g}(\mathrm{Q}, \mathrm{S})=\mathrm{Q}(\mathrm{x}, \mathrm{y}) * \mathrm{~S}(\mathrm{x}, \mathrm{y})$

$\mathrm{g}(\mathrm{Q}, \mathrm{S})=\sum_{u}^{a}=-a \sum_{v}^{b}=-b Q(u, v) S(x-u, y-v)$

$\mathrm{A}(\mathrm{x}, \mathrm{y})=\mathrm{g}(\mathrm{Q}, \mathrm{S})=\sum_{u}^{a}=-a \sum_{v}^{b}=-b Q(u, v) S(x-$

$u, y-v) \ldots \ldots \ldots . .1$

$\mathrm{G}(\mathrm{Q}, \mathrm{T})=\mathrm{Q}(\mathrm{x}, \mathrm{y}) * \mathrm{~T}(\mathrm{x}, \mathrm{y})$ 
$\mathrm{g}(\mathrm{Q}, \mathrm{T})=\sum_{u}^{a}=-a \sum_{v}^{b}=-b Q(u, v) T(x-u, y-v)$ $\mathrm{B}(\mathrm{x}, \mathrm{y})=\mathrm{g}(\mathrm{Q}, \mathrm{T})=\sum_{u}^{a}=-a \sum_{v}^{b}=-b Q(u, v) T(x-$ $u, y-v) \ldots . . . . . .2$

From equation 1 and 2

$\mathrm{X}(\mathrm{x}, \mathrm{y})=\mathrm{A}(\mathrm{x}, \mathrm{y}) * \mathrm{~B}(\mathrm{x}, \mathrm{y})$

\section{WGIEA}

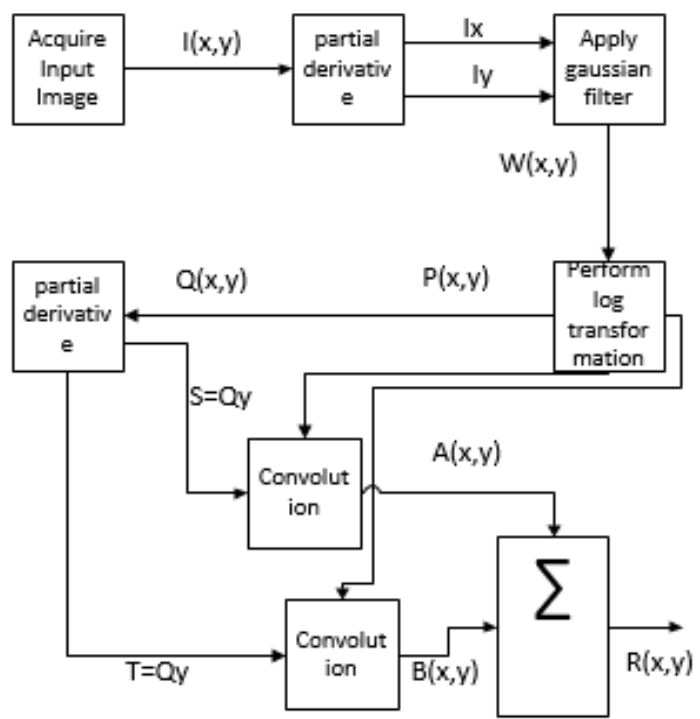

Figure 4

The acquire RGB image $I(\mathrm{x}, \mathrm{y})$ where we have to calculate $I_{\mathrm{x}}, \& I_{\mathrm{y}}$ from the Image $I(\mathrm{x}, \mathrm{y})$.

For the Further precedence:

$\left[\begin{array}{l}I x \\ I y\end{array}\right]=\left[\begin{array}{c}\frac{\partial I(x, y)}{\partial x} \\ \frac{\partial I(x, y)}{\partial y}\end{array}\right]$

Noise Removal: $W=\frac{1}{\sqrt{\sqrt{(2 \pi \sigma)}}} e^{-\frac{\left(I x^{2}+I y^{2}\right)}{2 \sigma}}$

$\mathrm{P}=\log (\mathrm{W},(\mathrm{x}, \mathrm{y}))$

$\mathrm{Q}=\mathrm{P}$

$\left[\begin{array}{l}Q x \\ Q y\end{array}\right]=\left[\begin{array}{l}\frac{\partial Q(x, y)}{\partial x} \\ \frac{\partial Q(x, y)}{\partial y}\end{array}\right]$

$S=Q(x)$

$T=Q(y)$

$\mathrm{g}(\mathrm{Q}, \mathrm{S})=\mathrm{Q}(\mathrm{x}, \mathrm{y}) * \mathrm{~S}(\mathrm{x}, \mathrm{y})$

$\mathrm{g}(\mathrm{Q}, \mathrm{S})=\sum_{u}^{a}=-a \sum_{v}^{b}=-b Q(u, v) S(x-u, y-v)$

$\mathrm{A}(\mathrm{x}, \mathrm{y})=\mathrm{g}(\mathrm{Q}, \mathrm{S})=\sum_{u}^{a}=-a \sum_{v}^{b}=-b Q(u, v) S(x-$

$u, y-v) \ldots \ldots \ldots . .1$

$\mathrm{G}(\mathrm{Q}, \mathrm{T})=\mathrm{Q}(\mathrm{x}, \mathrm{y}) * \mathrm{~T}(\mathrm{x}, \mathrm{y})$

$\mathrm{g}(\mathrm{Q}, \mathrm{T})=\sum_{u}^{a}=-a \sum_{v}^{b}=-b Q(u, v) T(x-u, y-v)$

$\mathrm{B}(\mathrm{x}, \mathrm{y})=\mathrm{g}(\mathrm{Q}, \mathrm{T})=\sum_{u}^{a}=-a \sum_{v}^{b}=-b Q(u, v) T(x-$

$u, y-v) \ldots . . . . .2$

From equation 1 and 2

$\mathrm{X}(\mathrm{x}, \mathrm{y})=\mathrm{A}(\mathrm{x}, \mathrm{y})+\mathrm{B}(\mathrm{x}, \mathrm{y})$

\section{$\underline{\text { WGIEM }}$}

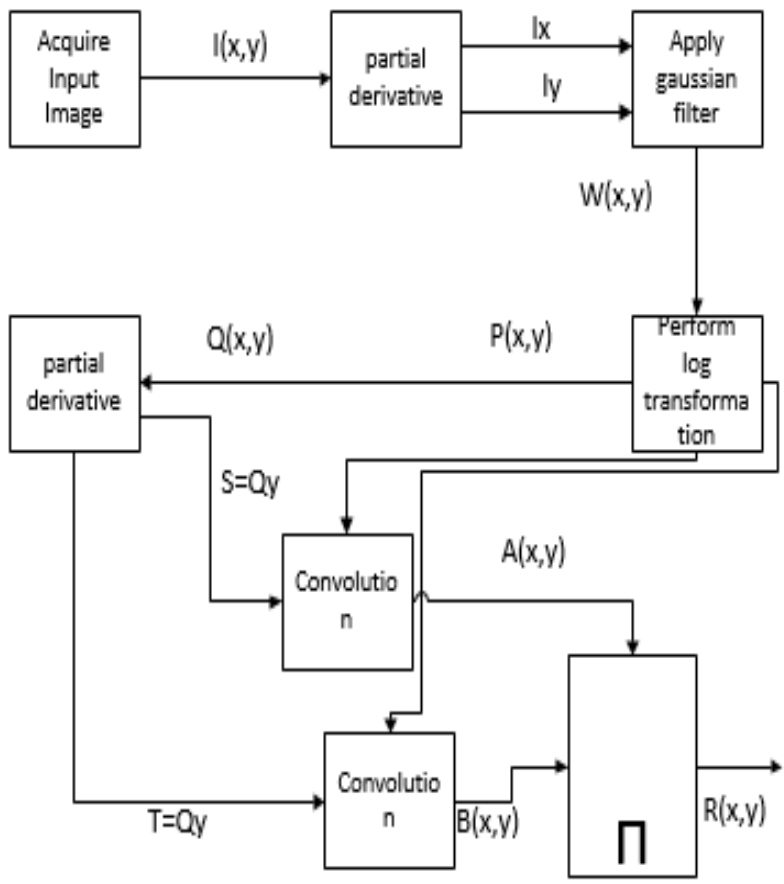

Figure 5

The acquire RGB image $I(\mathrm{x}, \mathrm{y})$ where we have to calculate $I_{\mathrm{x}}, \& I_{\mathrm{y}}$ from the Image $I(\mathrm{x}, \mathrm{y})$.

For the Further precedence:

$\left[\begin{array}{l}I x \\ I y\end{array}\right]=\left[\begin{array}{l}\frac{\partial I(x, y)}{\partial x} \\ \frac{\partial I(x, y)}{\partial y}\end{array}\right]$

Noise Removal: $W=\frac{1}{\sqrt{\sqrt{(2 \pi \sigma)}}} e^{-\frac{\left(I x^{2}+I y^{2}\right)}{2 \sigma}}$

$\mathrm{P}=\log (\mathrm{W},(\mathrm{x}, \mathrm{y}))$

$\mathrm{Q}=\mathrm{P}$

$\left[\begin{array}{l}Q x \\ Q y\end{array}\right]=\left[\begin{array}{c}\frac{\partial Q(x, y)}{\partial x} \\ \frac{\partial Q(x, y)}{\partial y}\end{array}\right]$

$S=Q(x)$

$T=Q(y)$

$\mathrm{g}(\mathrm{Q}, \mathrm{S})=\mathrm{Q}(\mathrm{x}, \mathrm{y}) * \mathrm{~S}(\mathrm{x}, \mathrm{y})$

$\mathrm{g}(\mathrm{Q}, \mathrm{S})=\sum_{u}^{a}=-a \sum_{v}^{b}=-b Q(u, v) S(x-u, y-v)$

$\mathrm{A}(\mathrm{x}, \mathrm{y})=\mathrm{g}(\mathrm{Q}, \mathrm{S})=\sum_{u}^{a}=-a \sum_{v}^{b}=-b Q(u, v) S(x-$

$u, y-v) \ldots \ldots \ldots . .1$

$\mathrm{G}(\mathrm{Q}, \mathrm{T})=\mathrm{Q}(\mathrm{x}, \mathrm{y}) * \mathrm{~T}(\mathrm{x}, \mathrm{y})$

$\mathrm{g}(\mathrm{Q}, \mathrm{T})=\sum_{u}^{a}=-a \sum_{v}^{b}=-b Q(u, v) T(x-u, y-v)$

$\mathrm{B}(\mathrm{x}, \mathrm{y})=\mathrm{g}(\mathrm{Q}, \mathrm{T})=\sum_{u}^{a}=-a \sum_{v}^{b}=-b Q(u, v) T(x-$

$u, y-v) \ldots . . . . .2$

From equation 1 and 2

$\mathrm{X}(\mathrm{x}, \mathrm{y})=\mathrm{A}(\mathrm{x}, \mathrm{y}) * \mathrm{~B}(\mathrm{x}, \mathrm{y})$ 


\section{SIMULATION AND RESULTS}

Matlab R2017a. tool is used for simulation and results. For verification and validation of these 4 proposed algorithmsfor edge detection is apply on 9 different kind of images. The results of these algorithms are shown in table 2 which shows that GIEA and WOGIEA algorithm is given most appropriate results as compare to GIEM and WOGIEM.

\begin{tabular}{|c|c|c|c|c|c|}
\hline \multirow{2}{*}{$\begin{array}{c}\mathrm{S} \\
\mathrm{r} \\
\# .\end{array}$} & \multirow[t]{2}{*}{$\begin{array}{l}\text { TEST Acquired } \\
\text { IMAGEs }\end{array}$} & \multicolumn{2}{|c|}{$\begin{array}{l}\text { Edge Detection using Proposed GIE } \\
\text { Methods }\end{array}$} & \multicolumn{2}{|c|}{$\begin{array}{c}\text { Edge Detection using Proposed WOGIE } \\
\text { Methods }\end{array}$} \\
\hline & & GIEA & GIEM & WOGIEA & WOGIEM \\
\hline 1. & & 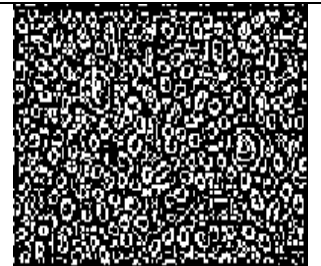 & 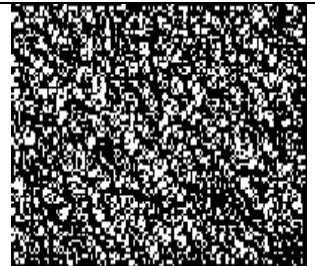 & 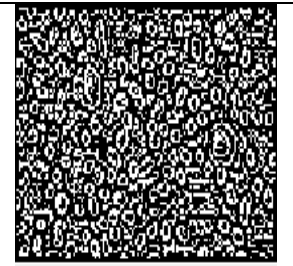 & $\begin{array}{l}3 \\
7 \times 3 \\
4\end{array}$ \\
\hline 2. & & 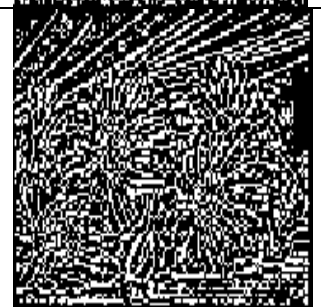 & 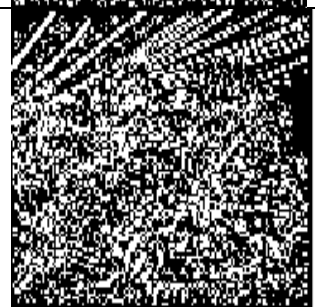 & 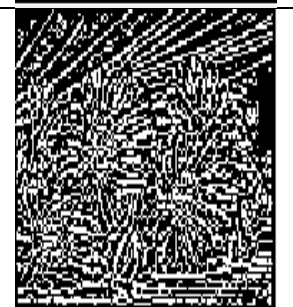 & 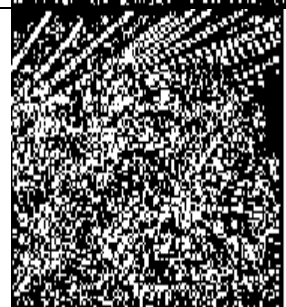 \\
\hline 3. & & 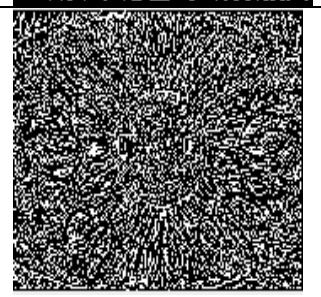 & 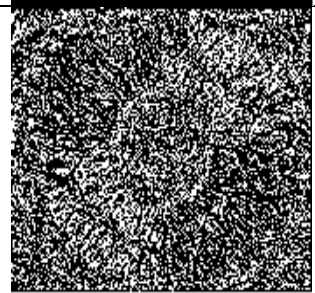 & 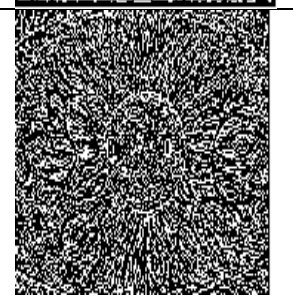 & 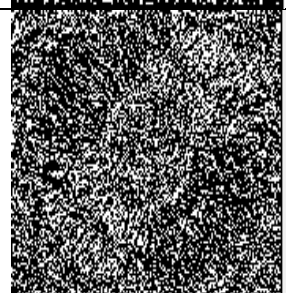 \\
\hline 4. & & 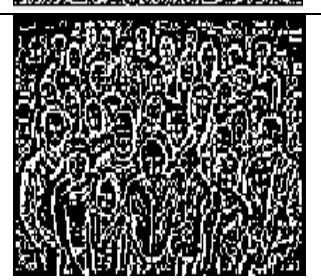 & 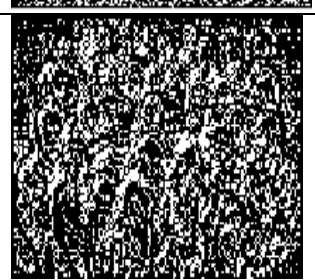 & 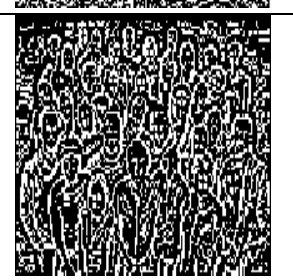 & 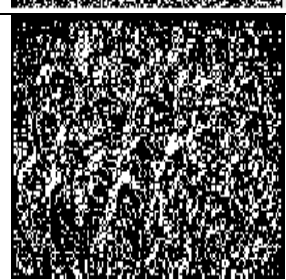 \\
\hline 5. & & 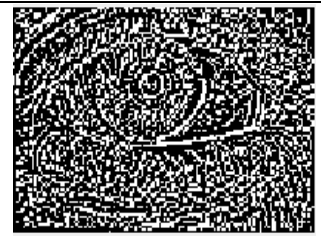 & 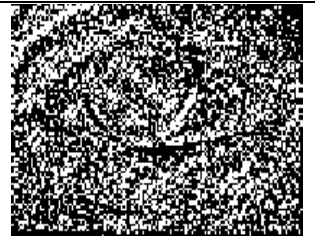 & 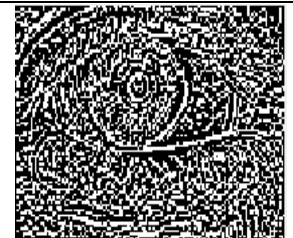 & 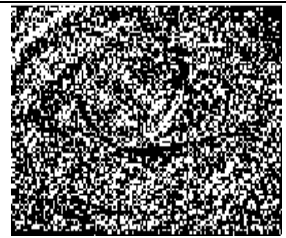 \\
\hline 6. & & 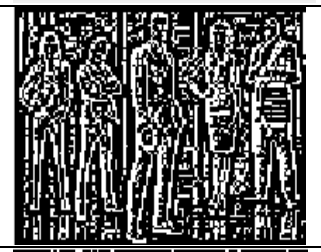 & 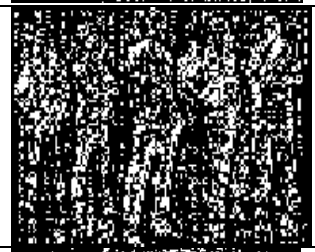 & 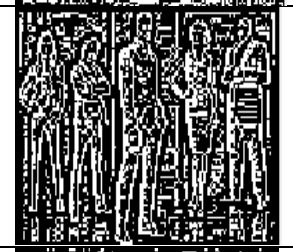 & 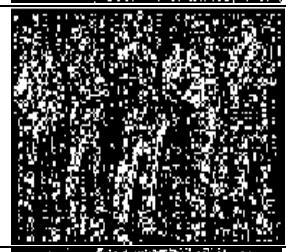 \\
\hline 7. & & 4 & & & \\
\hline & & 6/3) & 9 & \% & $\begin{array}{l}6 \\
3 \\
3\end{array}$ \\
\hline
\end{tabular}



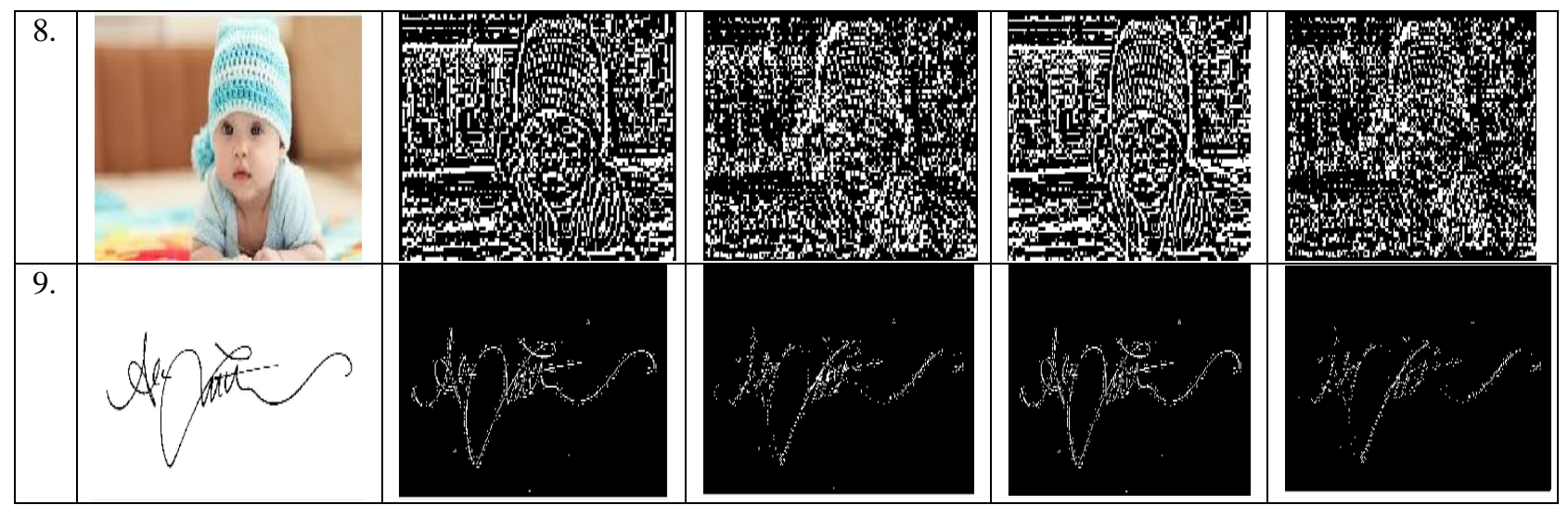

Table 1: Results of the simulations

\section{CONCLUSION}

Edge detection is providing foundation to medical image processing applications as well as armed forces applications [18]. In this research article 4 different methods for edge detection are introduced. These are as following:

$$
\text { - } \text { - GIEA }
$$

\section{REFERENCES}

[1] A. Azeroual and K. Afdel, "Fast Image Edge Detection based on Faber Schauder Wavelet and Otsu Threshold," Heliyon, vol. 3, no. 12, p. e00485, 2017.

[2] E. Dong, Y. Zhao, X. Yu, J. Zhu, and C. Chen, "An Improved NMS-Based Adaptive Edge Detection Method and Its FPGA Implementation," vol. 2016, 2016.

[3] D. Ziou and S. Tabbone, "Edge detection techniques - an overview," Pria, no. October, pp. 1-41, 1998.

[4] M. A. Otair, A. N. A. Alnajjar, and A. Odat, "An Automatic Method for Edge Detection Evaluation based on Semi-Optimal Edge Detector," Indian J. Sci. Technol., vol. 9, no. 39, pp. 0-8, 2016.

[5] P. Selvakumar and S. Hariganesh, "The performance analysis of edge detection algorithms for image processing," in 2016 International Conference on Computing Technologies and Intelligent Data Engineering (ICCTIDE'16), 2016, pp. 1-5.

[6] T. Nagasankar and B. Ankaryarkanni, "Performance analysis of edge detection algorithms on various image types," Indian J.
- WOGIEA

- WOGIEM.

The simulation of all these algorithms is done in Matlab R2017a.The results of simulation are shown in table 2. Experimental result shows that GIEA algorithm gives batter results as compare to GIEM, WOGIEA and WOGIEM.

Sci. Technol., vol. 9, no. 21, 2016.

[7] U. G. Nevagi, "Edge Detection Techniques: A Survey," Int. J. Innov. Res. Dev., vol. 5, no. 2, pp. 274-281, 2016.

[8] T. Nadu, "Sobel edge detection technique implementation for image steganography analysis .," pp. 1-7, 2017.

[9] I. Journal, O. F. Engineering, E. Analysis, O. F. Edge, and D. Algorithms, "PERFORMANCE EVALUATION AND EFFECTIVE ANALYSIS OF EDGE," vol. 6, no. 6 , pp. 282-286, 2017.

[10] L. Huang, X. Yu, and X. Zuo, "Edge Detection in UAV Remote Sensing Images Using the Method Integrating Zernike Moments with Clustering Algorithms," Int. J. Aerosp. Eng. Geoinf., vol. 2017, no. 2006, 2017.

[11] Y. Icer and M. Turk, "Implementation of Mainly Used Edge Detection Algorithms on FPGA," Ijamec, vol. 4, no. Special Issue, pp. 352-358, 2016.

[12] L. Liu, F. Liang, J. Zheng, D. He, and J. Huang, "Ship infrared image edge detection based on an improved adaptive Canny algorithm," Int. J. Distrib. Sens. Networks, vol. 14, no. 3, 2018. 
[13] R. Al-jarrah, M. Al-jarrah, and H. Roth, "A Novel Edge Detection Algorithm for Mobile Robot Path Planning," Hindawi J. Robot., vol. 2018, pp. 1-12, 2018.

[14] J. Cao, L. Chen, M. Wang, and Y. Tian, "Implementing a Parallel Image Edge Detection Algorithm Based on the OtsuCanny Operator on the Hadoop Platform," Comput. Intell. Neurosci., vol. 2018, 2018.

[15] Z. W. Htet, K. Z. Ye, V. D. Koldaev, V. G. Dorogov, and N. L. Aung, "The edges detection in images using the clustering algorithm," in 2017 IEEE Conference of Russian Young Researchers in Electrical and Electronic Engineering (EIConRus), 2017, pp. 609-612.

[16] S. Z. J. S. S. C. H. H. A. B. Wenguan Wang, "Salient Object Detection With Pyramid Attention and Salient Edges," in Conference on Computer Vision and Pattern Recognition (CVPR), 2019.
[17] C. Li, C. Guo, W. Ren, R. Cong, J. Hou, S. Kwong and D. Tao, "An Underwater Image Enhancement Benchmark Dataset and Beyond," IEEE Transactions on Image Processing, vol. 29, pp. 4376 - 4389, 28 November 2019.

[18] S. M. a. S. M. Kalyan Kumar Jena, "An Algorithmic Approach Based on CMS Edge Detection Technique for the Processing of Digital Images," in Examining Fractal Image Processing and Analysis, 2020, p. 21.

[19] Z. Zheng, B. Zha, H. Yuan, Y. Xuchen, Y. Gao and H. Zhang, "Adaptive Edge Detection Algorithm Based on Improved Grey Prediction Model," IEEE Access, vol. 8, pp. 102165 - 102176, 01 June 2020.

[20] Yuhi Kaihoko, Phan Xuan Tan, Eiji Kamioka, "Identification of photo-taking behaviors using optical flow vector," International Journal of Advanced Trends in Computer Science and Engineering, vol. 8, pp. 306 312, 2019. 